\title{
Synthesis of symmetrical and unsymmetrical 1,3,4-oxadiazoles and their interconversion to 1,3,4-thiadiazoles and 1,2,4-triazoles
}

\section{Venkatapuram Padmavathi,* Gali Sudhakar Reddy, Annaji Venkata Nagendra Mohan, and Konda Mahesh}

Department of Chemistry, Sri Venkateswara University, Tirupati 517 502, Andhra Pradesh, India

E-mail:vkpuram2001@yahoo.com

\begin{abstract}
A new class of symmetrical and unsymmetrical 1,3,4-oxadiazoles was prepared. Interconversion of oxadiazoles to thiadiazoles and triazoles was effected in the presence of appropriate nucleophiles.
\end{abstract}

Keywords: 1,3,4-Oxadiazoles, 1,3,4-thiadiazoles, 1,2,4-triazoles, cyclocondensation

\section{Introduction}

Amongst five membered aromatic heterocycles oxadiazoles, thiadiazoles and triazoles have attracted significant interest in medicinal and pesticide chemistry and polymer and material science. Symmetrical and unsymmetrical 1,3,4-oxadiazoles are biologically versatile compounds displaying a variety of biological effects which include anti-inflammatory, ${ }^{1}$ antifungal, ${ }^{2}$ antiparasitic, ${ }^{3}$ and antimicrobial activities. ${ }^{4}$ The widespread use of 1,3,4-oxadiazoles as a scaffold in medicinal chemistry established this moiety as a member of the privileged structures class. One of the popular methods for the synthesis of 1,3,4-oxadiazoles involves cyclization of diacylhydrazines prepared from the reaction of acyl chlorides and hydrazine. Several cyclodehydrating agents such as $\mathrm{Et}_{2} \mathrm{O} \cdot \mathrm{BF}_{3}{ }^{5}$ 1,1,1,3,3,3-hexamethyldisilazane, ${ }^{6}$ triflic anhydride, ${ }^{7}$ phosphorus pentoxide, ${ }^{8}$ polyphosphoric acid, ${ }^{9}$ thionyl chloride, ${ }^{10}$ phosphorus oxychloride ${ }^{11}$ and sulfuric acid $^{12}$ have been used. One-pot synthesis of 1,3,4-oxadiazoles from hydrazine with carboxylic acids have also been reported. ${ }^{13}$ 2,5-Disubstituted 1,3,4-thiadiazoles possess various biological properties such as antitumor, ${ }^{14}$ anticonvulsant, ${ }^{15}$ antibacterial, ${ }^{15}$ antifungal, ${ }^{16}$ antiinflammatory, ${ }^{17}$ antihypertensive, ${ }^{18}$ anaesthetic ${ }^{19}$ and cardiotonic activities. ${ }^{20}$ Most frequently used methods for the synthesis of thiadiazoles include the reaction of acylthiosemicarbazides with acidic reagents such as concentrated sulfuric acid, ${ }^{21}$ acetic acid, ${ }^{22}$ methanesulfonic acid, ${ }^{23}$ $85 \%$ phosphoric acid. ${ }^{24}$ In addition, triazoles have found wide use in medicinal chemistry as 
common structural motifs acting as peptidomimetic moieties and as hydrogen bond acceptors. ${ }^{25}$ They possess important pharmacological activities such as antifungal and antiviral. Examples of antifungal drugs are fluconazole, ${ }^{26}$ itraconazole $^{27}{ }^{27}$ avuconazole, ${ }^{28}$ voriconazole, ${ }^{29}$ and posaconazole. ${ }^{30}$ One of the synthetic methods for the preparation of triazoles involves the use of $N, N$-dimethyl formamide dimethyl acetals. ${ }^{31}$ The present communication deals with the synthesis of hitherto unknown symmetrical and unsymmetrical 1,3,4-oxadiazoles and their interconversion into thiadiazoles and triazoles in the presence of appropriate reagents.

\section{Results and Discussion}

In order to synthesize the target molecules, we have used the acid hydrazides of arylsulfonylacetic acid $\mathbf{5}$ and arylmethanesulfonylacetic acid $\mathbf{6}$ as synthetic intermediates. The $\mathbf{5}$ and 6 were prepared from the corresponding acids on esterification followed by treatment with hydrazine hydrate. The symmetrical 1,3,4-oxadiazoles, 2,5-bis(arylsulfonylmethyl)-1,3,4oxadiazoles 7 were prepared by the cyclocondensation of arylsulfonylacetic acid $\mathbf{1}$ with $\mathbf{5}$ in the presence of phosphorus oxychloride. Similarly, 2,5-bis(benzylsulfonylmethyl)-1,3,4-oxadiazoles 8 were obtained by the reaction of benzylsulfonylacetic acid (2) with 6 in the presence of phosphorus oxychloride (see Scheme 1 and Mechanism 1). The ${ }^{1} \mathrm{H}$ NMR spectrum of 7a displayed a singlet at $4.18 \mathrm{ppm}$ which was assigned to methylene protons. The ${ }^{13} \mathrm{C} \mathrm{NMR}$ spectrum of 7a exhibited a signal at $55.1 \mathrm{ppm}$ for methylene carbon apart from signals due to aromatic carbons. However, 8a showed two singlets at 4.07 and $4.59 \mathrm{ppm}$ for the methylene protons flanked between sulfonyl and heterocyclic ring and benzylic protons. The ${ }^{13} \mathrm{C}$ NMR spectrum of 8a displayed two signals at 55.4 and $57.8 \mathrm{ppm}$ due to methylene carbons. The signal which appears in the downfield region was assigned to the benzylic carbon. Thus, the highly symmetric nature of the compounds 7 and 8 was confirmed by ${ }^{1} \mathrm{H}$ and ${ }^{13} \mathrm{C}$ NMR spectra. On the other hand, 2-(benzylsulfonylmethyl)-5-(arylsulfonylmethyl)-1,3,4-oxadiazoles 9 were obtained by the reaction of 1 with 6 (Method A) or 2 with 5 (Method B) in the presence of phosphorus oxychloride. The identity of compound 9 prepared in two methods was confirmed by TLC and ${ }^{1} \mathrm{H}$ NMR spectra. The ${ }^{1} \mathrm{H}$ NMR spectrum of 9a displayed three singlets at 3.94, 4.18, $4.46 \mathrm{ppm}$ which were assigned to the methylene protons present between the sulfonyl and the heterocyclic, the aryl and the sulfonyl and the arylsulfonyl and the heterocyclic moieties. The ${ }^{13} \mathrm{C} \mathrm{NMR}$ spectrum of 9a showed three signals at 57.7, 53.5 and 58.9 due to the benzylic carbon and the methylene carbons present between the sulfonyl group and the heterocyclic ring, and the arylsulfonyl group and the heterocyclic ring, respectively apart from signals due to aromatic carbons. 

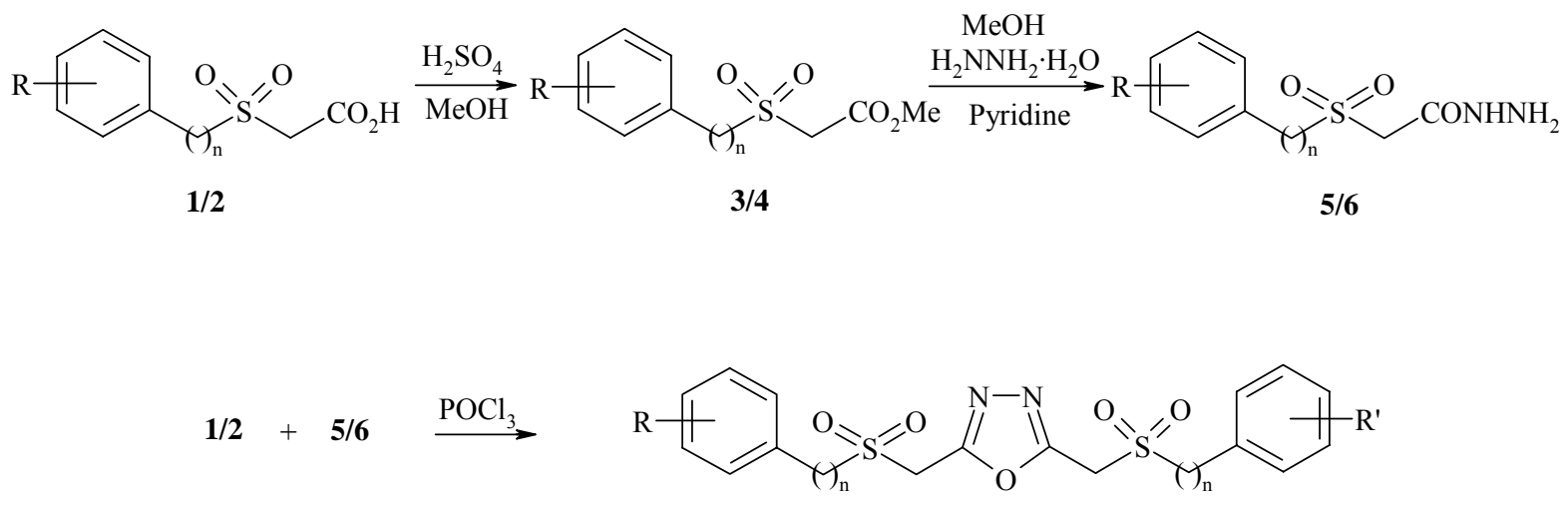

$1,3,5,7 \mathrm{n}=0$

2,4,6,8 $n=1$

$7 / 8$

$1+6 \underset{\mathrm{POCl}_{3}}{\stackrel{\text { Method A }}{\longrightarrow}}$
a: $\mathrm{R}=\mathrm{R}^{\prime}=\mathrm{H}$
b: $\mathrm{R}=\mathrm{R}^{\prime}=4-\mathrm{Me}$
c: $\mathrm{R}=\mathrm{R}^{\prime}=4-\mathrm{Cl}$

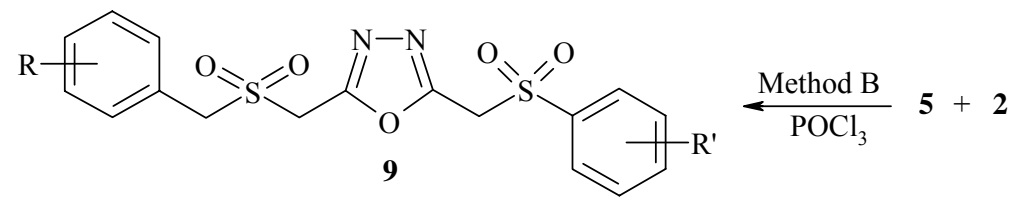

\section{Scheme 1}

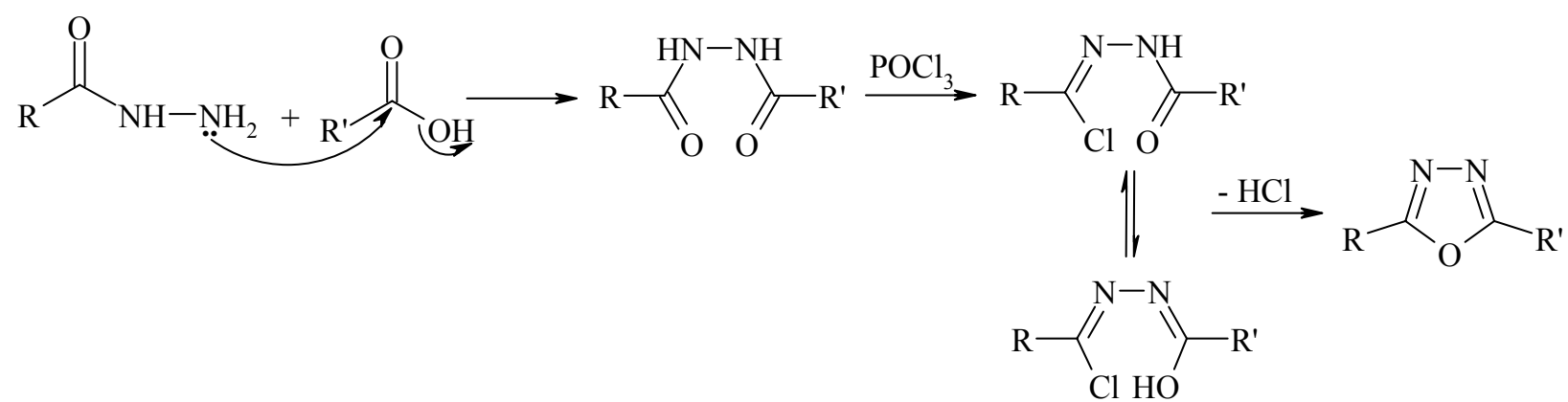

\section{Mechanism 1}

Replacement of $-\mathrm{O}-$ by $-\mathrm{S}-$ or $-\mathrm{NH}-$ in heterocycles was reported viz., Bordners ${ }^{32}$ preparation of pyrroles from furan and the transformation of epoxides to episulfides by the action of thiocyanates or thiourea. ${ }^{33}$ The compounds 7, 8 and 9 were treated with two fold excess thiourea in tetrahydrofuran. The reaction mixture after workup gave a solid which was identified as 2,5-bis(arylsulfonylmethyl)-1,3,4-thiadiazole $\quad \mathbf{1 0}, \quad 2,5$-bis(benzylsulfonylmethyl)-1,3,4thiadiazole $\mathbf{1 1}$ and 2-(benzylsulfonylmethyl)-5-(arylsulfonylmethyl)-1,3,4-thiadiazole 12, respectively. The probable mechanism involves the formation of thiouronium salt which undergoes rearrangement to form mesomeric oxouronium salt via oxathiadiazepine derivative. Further, ring closure of oxouronium salt led to thiadiazole by the elimination of urea (see 
Scheme 2 and Mechanism 2). On the other hand, treatment of 7, 8 and 9 with excess hydrazine hydrate gave 3,5-bis(arylsulfonylmethyl)-4-amino-1,2,4-triazole 13, 3,5bis(benzylsulfonylmethyl)-4-amino-1,2,4-triazole $\mathbf{1 4}$ and 3-(benzylsulfonylmethyl)-5(arylsulfonylmethyl)-4-amino-1,2,4-triazole 15, respectively. The reaction may proceed by the nucleophilic attack of hydrazine on $\alpha$-carbon atom of oxadiazole ring followed by dehydration (see Mechanism 3). The ${ }^{1} \mathrm{H}$ NMR spectra of 10a, 11a, 13a and 14a exhibited a singlet at 4.21, 4.12, 4.22 and $4.14 \mathrm{ppm}$ for methylene protons present between sulfonyl and heterocyclic moieties. Apart from this, compound 11a and 14a displayed another singlet at 4.65 and $4.64 \mathrm{ppm}$ for benzylic protons. On the other hand, three singlets at 3.93, 4.25 and $4.52 \mathrm{ppm}$ were observed in 12a while at 3.92, 4.21 and 4.50 in 15a due to methylene protons present between sulfonyl and heterocyclic ring, aryl and sulfonyl and arylsulfonyl and heterocyclic ring. The structure of these compounds was further confirmed by ${ }^{13} \mathrm{C}$ NMR spectra.
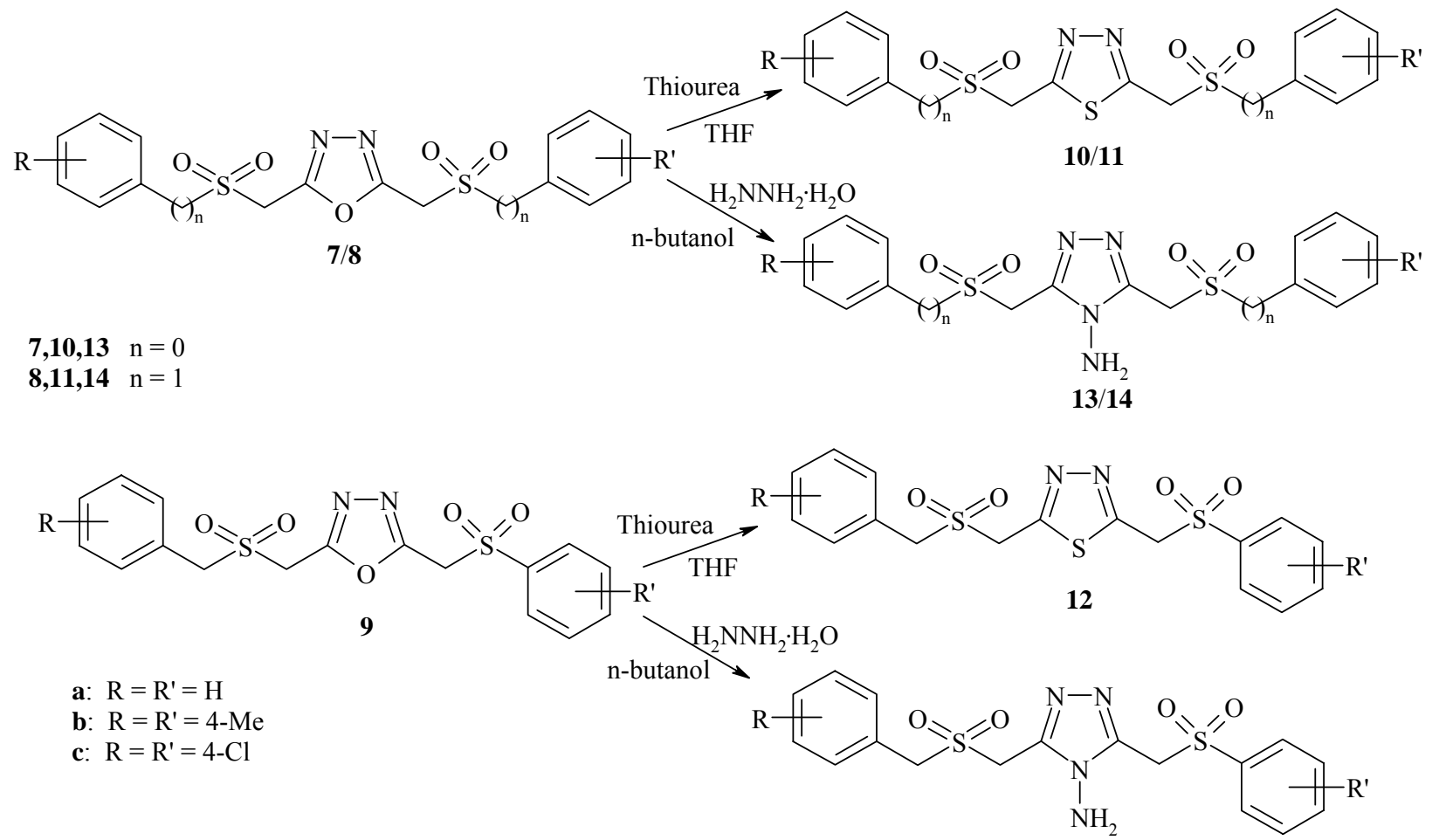

15

\section{Scheme 2}




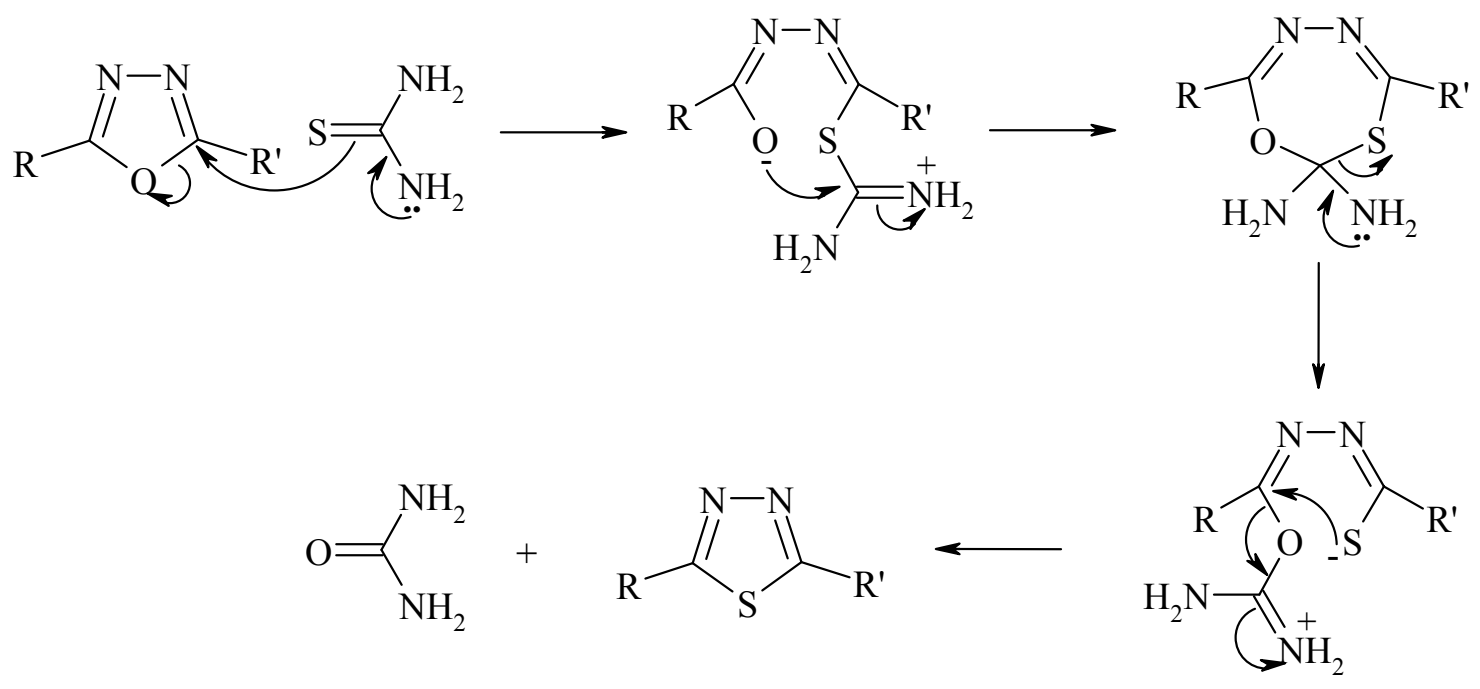

\section{Mechanism 2}

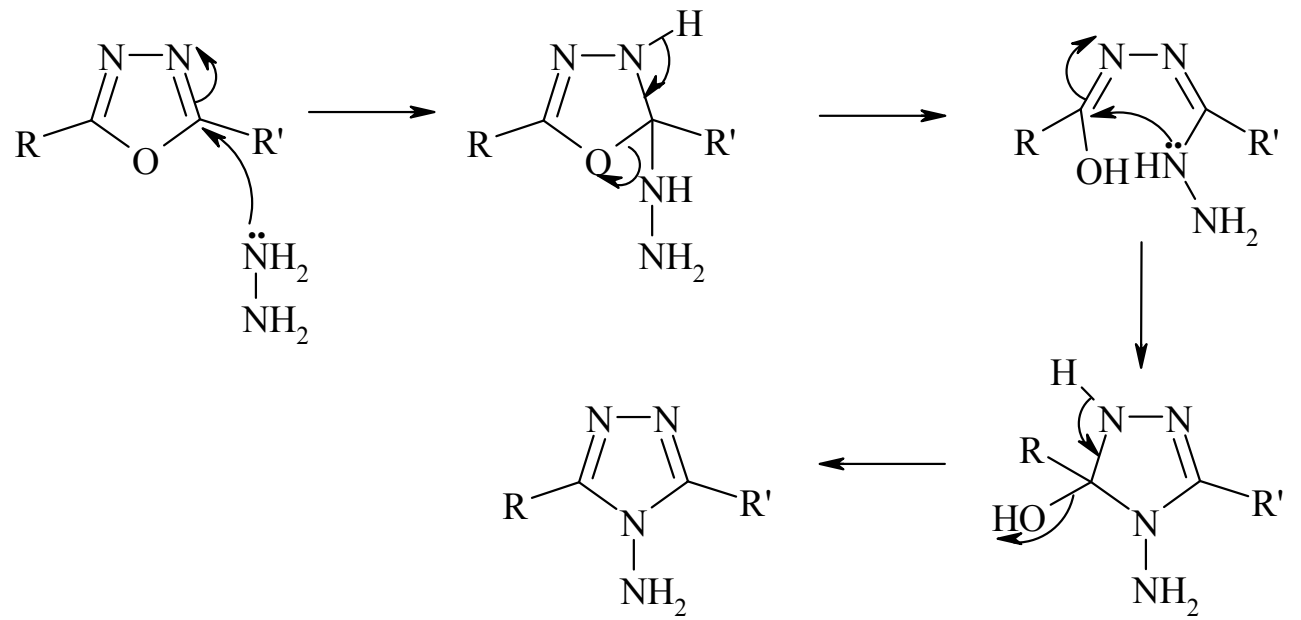

\section{Mechanism 3}

\section{Conclusions}

A new class of symmetrical and unsymmetrical 1,3,4-oxadiazoles was prepared adopting simple and versatile methodology. Interconversion of oxadiazoles to thiadiazoles and triazoles was effected in the presence of appropriate nucleophiles. 


\section{Experimental Section}

General Procedures. Melting points were determined in open capillaries on a Mel-Temp apparatus and are uncorrected. The purity of the compounds was checked by TLC (silica gel H, BDH, ethyl acetate/hexane, 1:3). The IR spectra were recorded on a Thermo Nicolet IR 200 FTIR spectrometer as $\mathrm{KBr}$ pellets and the wave numbers were given in $\mathrm{cm}^{-1}$. The ${ }^{1} \mathrm{H} \mathrm{NMR}$ spectra were recorded in $\mathrm{CDCl}_{3} / \mathrm{DMSO}-d_{6}$ on a Varian EM-360 spectrometer $(300 \mathrm{MHz})$. The ${ }^{13} \mathrm{C}$ NMR spectra were recorded in $\mathrm{CDCl}_{3} / \mathrm{DMSO}-d_{6}$ on a Varian VXR spectrometer operating at 75.5 MHz. All chemical shifts are reported in $\delta(\mathrm{ppm})$ using TMS as an internal standard. The microanalyses were performed on a Perkin-Elmer $240 \mathrm{C}$ elemental analyzer. The starting compounds arylsulfonylacetic acid (1) arylmethanesulfonylacetic acid (2), arylsulfonylacetic acid hydrazide (5) and arylmethanesulfonylacetic acid hydrazide (6) were prepared by the literature procedure. ${ }^{34}$

\section{2,5-Bis(phenylsulfonylmethyl)-1,3,4-oxadiazole (7a). Typical procedure}

A mixture of $5 \mathbf{a}(2.14 \mathrm{~g}, 10 \mathrm{mmol}), 1 \mathrm{a}(2.00 \mathrm{~g}, 10 \mathrm{mmol})$ and $\mathrm{POCl}_{3}(7 \mathrm{~mL})$ was heated under reflux for 5-6 h. The excess $\mathrm{POCl}_{3}$ was removed under reduced pressure and the residue was poured onto crushed ice. The resulting precipitate was filtered, washed with saturated sodium bicarbonate solution and then with water, dried and recrystallized from ethanol to get 7a. Yield 2.83 g (75\%); white solid; m. p. $119-121{ }^{\circ} \mathrm{C} ;{ }^{1} \mathrm{H}$ NMR (300 MHz, DMSO- $\left.d_{6}\right): \delta=4.18$ (s, 4H, $\left.\mathrm{CH}_{2}\right), 7.18-7.49(\mathrm{~m}, 10 \mathrm{H}, \mathrm{Ar}-\mathrm{H}) ;{ }^{13} \mathrm{C}$ NMR $\left(300 \mathrm{MHz}, \mathrm{DMSO}-d_{6}\right): \delta=55.1\left(\mathrm{CH}_{2}\right), 162.3(\mathrm{C}-2 \&$ $\mathrm{C}-5), 126.4,128.9,129.7,134.3$ (aromatic carbons); IR $(\mathrm{KBr}): v=1636(\mathrm{C}=\mathrm{N}), 1328,1125$ $\left(\mathrm{SO}_{2}\right)$; Anal. Calcd. for $\mathrm{C}_{16} \mathrm{H}_{14} \mathrm{~N}_{2} \mathrm{O}_{5} \mathrm{~S}_{2}$ (378.42): C, 50.78; H, 3.73; N, 7.40. Found: C, 50.85; H, $3.71 ; \mathrm{N}, 7.48$.

2,5-Bis(4-methylphenylsulfonylmethyl)-1,3,4-oxadiazole (7b). Yield $3.08 \mathrm{~g}$ (76\%); white solid; m. p. $145-147{ }^{\circ} \mathrm{C} ;{ }^{1} \mathrm{H}$ NMR (300 MHz, DMSO- $\left.d_{6}\right): \delta=2.25$ (s, 6H, Ar-CH $), 4.14$ (s, 4H, $\left.\mathrm{CH}_{2}\right)$, 7.24-7.52 (m, 8H, Ar-H); ${ }^{13} \mathrm{C}$ NMR (300 MHz, DMSO- $\left.d_{6}\right): \delta=21.2\left(\mathrm{Ar}_{-} \mathrm{CH}_{3}\right), 54.4$ $\left(\mathrm{CH}_{2}\right), 162.7$ (C-2 \& C-5), 127.1, 127.6, 133.2, 134.4 (aromatic carbons); IR (KBr): $v=1625$ $(\mathrm{C}=\mathrm{N})$, 1323, $1132\left(\mathrm{SO}_{2}\right)$; Anal. Calcd. for $\mathrm{C}_{18} \mathrm{H}_{18} \mathrm{~N}_{2} \mathrm{O}_{5} \mathrm{~S}_{2}$ (406.48): C, 53.19; H, 4.46; N, 6.89. Found: C, 53.24; H, 4.48; N, 6.82 .

2,5-Bis(4-chlorophenylsulfonylmethyl)-1,3,4-oxadiazole (7c). Yield $3.57 \mathrm{~g}$ (80\%); white crystals; m. p. $162-164{ }^{\circ} \mathrm{C} ;{ }^{1} \mathrm{H}$ NMR $\left(300 \mathrm{MHz}, \mathrm{DMSO}-d_{6}\right): \delta=4.16\left(\mathrm{~s}, 4 \mathrm{H}, \mathrm{CH}_{2}\right), 7.42-7.78(\mathrm{~m}$, $8 \mathrm{H}, \mathrm{Ar}-\mathrm{H}) ;{ }^{13} \mathrm{C}$ NMR $\left(300 \mathrm{MHz}, \mathrm{DMSO}-d_{6}\right): \delta=54.9\left(\mathrm{CH}_{2}\right), 163.0(\mathrm{C}-2 \& \mathrm{C}-5), 126.7,129.3$, 134.7, 136.6 (aromatic carbons); IR $(\mathrm{KBr}): v=1628(\mathrm{C}=\mathrm{N}), 1332,1120\left(\mathrm{SO}_{2}\right)$; Anal. Calcd. for $\mathrm{C}_{16} \mathrm{H}_{12} \mathrm{Cl}_{2} \mathrm{~N}_{2} \mathrm{O}_{5} \mathrm{~S}_{2}$ (447.31): C, 42.96; H, 2.70; N, 6.26. Found: C, 42.90; H, 2.75; N, 6.32.

\section{2,5-Bis(benzylsulfonylmethyl)-1,3,4-oxadiazole (8a). Typical procedure}

A mixture of $6 \mathbf{a}(2.28 \mathrm{~g}, 10 \mathrm{mmol}), 2 \mathbf{a}(2.14 \mathrm{~g}, 10 \mathrm{mmol})$ and $\mathrm{POCl}_{3}(7 \mathrm{~mL})$ was heated under reflux for 4-5 h. The excess $\mathrm{POCl}_{3}$ was removed under reduced pressure and the residue was poured onto crushed ice. The resulting precipitate was filtered, washed with saturated sodium 
bicarbonate solution and then with water, dried and recrystallized from ethanol to get 8a. Yield 3.21 g (79\%); white solid; m. p. 195-197 ${ }^{\circ} \mathrm{C}$; ${ }^{1} \mathrm{H}$ NMR (300 MHz, DMSO- $\left.d_{6}\right): \delta=4.07$ (s, 4H, $\left.\mathrm{CH}_{2}\right), 4.59$ (s, 4H, Ar-CH 2$), 7.28-7.36(\mathrm{~m}, 10 \mathrm{H}, \mathrm{Ar}-\mathrm{H}) ;{ }^{13} \mathrm{C}$ NMR $\left(300 \mathrm{MHz}, \mathrm{DMSO}-d_{6}\right): \delta=$ $55.4\left(\mathrm{CH}_{2}\right), 57.8\left(\mathrm{Ar}-\mathrm{CH}_{2}\right), 160.8(\mathrm{C}-2 \& \mathrm{C}-5), 125.4,127.3,131.7,134.2$ (aromatic carbons); IR $(\mathrm{KBr}): v=1629(\mathrm{C}=\mathrm{N}), 1334,1137\left(\mathrm{SO}_{2}\right)$; Anal. Calcd. for $\mathrm{C}_{18} \mathrm{H}_{18} \mathrm{~N}_{2} \mathrm{O}_{5} \mathrm{~S}_{2}$ (406.48): C, 53.19; $\mathrm{H}$, 4.46; N, 6.89. Found: C, 53.26; H, 4.44; N, 6.93.

2,5-Bis(4-methylbenzylsulfonylmethyl)-1,3,4-oxadiazole (8b). Yield $3.56 \mathrm{~g}$ (82\%); white crystals; m. p. $222-224{ }^{\circ} \mathrm{C} ;{ }^{1} \mathrm{H}$ NMR $\left(300 \mathrm{MHz}, \mathrm{DMSO}-d_{6}\right): \delta=2.24\left(\mathrm{~s}, 6 \mathrm{H}, \mathrm{Ar}-\mathrm{CH}_{3}\right), 4.12(\mathrm{~s}$, $\left.4 \mathrm{H}, \mathrm{CH}_{2}\right), 4.62\left(\mathrm{~s}, 4 \mathrm{H}, \mathrm{Ar}-\mathrm{CH}_{2}\right), 7.30-7.39(\mathrm{~m}, 8 \mathrm{H}, \mathrm{Ar}-\mathrm{H}) ;{ }^{13} \mathrm{C}$ NMR $\left(300 \mathrm{MHz}, \mathrm{DMSO}-d_{6}\right): \delta=$ $21.7\left(\mathrm{Ar}-\mathrm{CH}_{3}\right), 55.1\left(\mathrm{CH}_{2}\right), 58.0\left(\mathrm{Ar}-\mathrm{CH}_{2}\right), 161.3(\mathrm{C}-2$ \& $\mathrm{C}-5), 125.1,129.4,131.8,134.1$ (aromatic carbons); IR $(\mathrm{KBr}): v=1626(\mathrm{C}=\mathrm{N}), 1336,1134\left(\mathrm{SO}_{2}\right)$; Anal. Calcd. for $\mathrm{C}_{20} \mathrm{H}_{22} \mathrm{~N}_{2} \mathrm{O}_{5} \mathrm{~S}_{2}$ (434.53): C, 55.28; H, 5.10; N, 6.45. Found: C, 55.32; H, 5.13; N, 6.49.

2,5-Bis(4-chlorobenzylsulfonylmethyl)-1,3,4-oxadiazole (8c). Yield $3.99 \mathrm{~g}$ (84\%); white solid; m. p. $230-232{ }^{\circ} \mathrm{C} ;{ }^{1} \mathrm{H}$ NMR $\left(300 \mathrm{MHz}, \mathrm{DMSO}-d_{6}\right): \delta=4.10\left(\mathrm{~s}, 4 \mathrm{H}, \mathrm{CH}_{2}\right), 4.64\left(\mathrm{~s}, 4 \mathrm{H}, \mathrm{Ar}-\mathrm{CH}_{2}\right)$, 7.40-7.45 (m, 8H, Ar-H); ${ }^{13} \mathrm{C}$ NMR (300 MHz, DMSO- $\left.d_{6}\right): \delta=55.6\left(\mathrm{CH}_{2}\right), 58.4\left(\mathrm{Ar}-\mathrm{CH}_{2}\right)$, 160.2 (C-2 \& C-5), 127.2, 129.1, 133.5, 134.0 (aromatic carbons); IR (KBr): $v=1622(\mathrm{C}=\mathrm{N})$, 1342, $1127\left(\mathrm{SO}_{2}\right)$; Anal. Calcd. for $\mathrm{C}_{18} \mathrm{H}_{16} \mathrm{Cl}_{2} \mathrm{~N}_{2} \mathrm{O}_{5} \mathrm{~S}_{2}$ (475.37): C, 45.48; H, 3.39; N, 5.89. Found: C, 45.43; H, 3.37; N, 5.94.

\section{2-(Benzylsulfonylmethyl)-5-(phenylsulfonylmethyl)-1,3,4-oxadiazole procedure}

(9a). Typical

Method A. A mixture of 1a $(2.00 \mathrm{~g}, 10 \mathrm{mmol})$, 6a $(2.28 \mathrm{~g}, 10 \mathrm{mmol})$ and $\mathrm{POCl}_{3}(7 \mathrm{~mL})$ was heated under reflux for 5-6 h. The excess $\mathrm{POCl}_{3}$ was removed under reduced pressure and the residue was poured onto crushed ice. The resulting precipitate was filtered, washed with saturated sodium bicarbonate solution and then with water, dried and recrystallized from ethanol to get 9a as a white solid. Yield $3.06 \mathrm{~g}(78 \%)$.

Method B. A mixture of 2a $(2.00 \mathrm{~g}, 10 \mathrm{mmol})$, 5a $(2.14 \mathrm{~g}, 10 \mathrm{mmol})$ and $\mathrm{POCl}_{3}(7 \mathrm{~mL})$ was heated under reflux for 4-5 h. The excess $\mathrm{POCl}_{3}$ was removed under reduced pressure and the residue was poured onto crushed ice. The resulting precipitate was filtered, washed with saturated sodium bicarbonate solution and then with water, dried and recrystallized from ethanol to get 9a. Yield $2.74 \mathrm{~g}(70 \%)$; white solid; m. p. $148-150{ }^{\circ} \mathrm{C}$; ${ }^{1} \mathrm{H}$ NMR $(300 \mathrm{MHz}$, $\left.\mathrm{CDCl}_{3}+\mathrm{DMSO}-d_{6}\right): \delta=3.94\left(\mathrm{~s}, 2 \mathrm{H}, \mathrm{SO}_{2}-\mathrm{CH}_{2}\right), 4.18\left(\mathrm{~s}, 2 \mathrm{H}, \mathrm{Ar}-\mathrm{CH}_{2}\right), 4.46\left(\mathrm{~s}, 2 \mathrm{H}, \mathrm{ArSO}_{2}-\mathrm{CH}_{2}\right)$, 7.23-7.41 (m, 10H, Ar-H); ${ }^{13} \mathrm{C}$ NMR (300 MHz, CDCl $\left.{ }_{3}+\mathrm{DMSO}-d_{6}\right): \delta=53.5\left(\mathrm{SO}_{2}-\mathrm{CH}_{2}\right), 57.7$ $\left(\mathrm{Ar}-\mathrm{CH}_{2}\right), 58.9\left(\mathrm{ArSO}_{2}-\mathrm{CH}_{2}\right), 157.3(\mathrm{C}-2), 159.1$ (C-5), 127.3, 127.4, 127.5, 128.2, 128.7, 131.7, 133.3, 135.7 (aromatic carbons); IR $(\mathrm{KBr}): v=1630(\mathrm{C}=\mathrm{N}), 1320,1135\left(\mathrm{SO}_{2}\right)$; Anal. Calcd. for $\mathrm{C}_{17} \mathrm{H}_{16} \mathrm{~N}_{2} \mathrm{O}_{5} \mathrm{~S}_{2}$ (392.45): C, 52.03; H, 4.11; N, 7.14. Found: C, 52.11; H, 4.14; N, 7.18.

2-(4-Methylbenzylsulfonylmethyl)-5-(4-methylphenylsulfonylmethyl)-1,3,4-oxadiazole (9b). Yield 3.19 g (76\%); white solid; m. p. $167-169{ }^{\circ} \mathrm{C} ;{ }^{1} \mathrm{H}$ NMR $\left(300 \mathrm{MHz}, \mathrm{DMSO}-d_{6}\right): \delta=2.21 \&$ 2.27 (s, 6H, Ar- $\left.\mathrm{CH}_{3}\right), 3.93\left(\mathrm{~s}, 2 \mathrm{H}, \mathrm{SO}_{2}-\mathrm{CH}_{2}\right), 4.22$ (s, 2H, Ar-CH$), 4.48$ (s, 2H, $\left.\mathrm{ArSO}_{2}-\mathrm{CH}_{2}\right)$, 7.26-7.34 (m, 8H, Ar-H); ${ }^{13} \mathrm{C}$ NMR (300 MHz, DMSO- $\left.d_{6}\right): \delta=21.5 \& 21.9\left(\mathrm{Ar}_{-} \mathrm{CH}_{3}\right), 53.2$ 
$\left(\mathrm{SO}_{2}-\mathrm{CH}_{2}\right), 57.4\left(\mathrm{Ar}-\mathrm{CH}_{2}\right), 59.1\left(\mathrm{ArSO}_{2}-\mathrm{CH}_{2}\right), 156.9(\mathrm{C}-2), 159.5$ (C-5), 125.4, 126.4, 128.1, 129.5, 130.7, 132.1, 133.7, 136.2 (aromatic carbons); IR $(\mathrm{KBr}): v=1637(\mathrm{C}=\mathrm{N}), 1334,1140$ $\left(\mathrm{SO}_{2}\right)$; Anal. Calcd. for $\mathrm{C}_{19} \mathrm{H}_{20} \mathrm{~N}_{2} \mathrm{O}_{5} \mathrm{~S}_{2}$ (420.50): C, 54.27; H, 4.79; N, 6.66. Found: C, 54.22; H, $4.76 ; \mathrm{N}, 6.60$.

2-(4-Chlorobenzylsulfonylmethyl)-5-(4-chlorophenylsulfonylmethyl)-1,3,4-oxadiazole (9c). Yield $3.31 \mathrm{~g}(72 \%)$; white crystals; m. p. $184-186{ }^{\circ} \mathrm{C}$; ${ }^{1} \mathrm{H}$ NMR $\left(300 \mathrm{MHz}, \mathrm{CDCl}_{3}+\mathrm{DMSO}-d_{6}\right)$ : $\delta=3.91\left(\mathrm{~s}, 2 \mathrm{H}, \mathrm{SO}_{2}-\mathrm{CH}_{2}\right), 4.20\left(\mathrm{~s}, 2 \mathrm{H}, \mathrm{Ar}-\mathrm{CH}_{2}\right), 4.51\left(\mathrm{~s}, 2 \mathrm{H}, \mathrm{ArSO}_{2}-\mathrm{CH}_{2}\right), 7.34-7.66(\mathrm{~m}, 8 \mathrm{H}$, $\mathrm{Ar}-\mathrm{H}) ;{ }^{13} \mathrm{C}$ NMR $\left(300 \mathrm{MHz}, \mathrm{CDCl}_{3}+\mathrm{DMSO}-d_{6}\right): \delta=53.8\left(\mathrm{SO}_{2}-\mathrm{CH}_{2}\right), 57.9\left(\mathrm{Ar}-\mathrm{CH}_{2}\right), 59.4$ $\left(\mathrm{ArSO}_{2}-\mathrm{CH}_{2}\right), 157.8$ (C-2), 158.7 (C-5), 125.7, 127.8, 128.3, 128.4, 132.0, 133.4, 134.4, 138.4 (aromatic carbons); IR $(\mathrm{KBr}): v=1625(\mathrm{C}=\mathrm{N}), 1345,1144\left(\mathrm{SO}_{2}\right) ;$ Anal. Calcd. for $\mathrm{C}_{17} \mathrm{H}_{14} \mathrm{Cl}_{2} \mathrm{~N}_{2} \mathrm{O}_{5} \mathrm{~S}_{2}$ (461.34): C, 44.26; H, 3.06; N, 6.07. Found: C, 44.32; H, 3.00; N, 6.14.

\section{2,5-Bis(phenylsulfonylmethyl)-1,3,4-thiadiazole (10a). Typical procedure}

In a sealed test tube, a mixture of 7a $(1.89 \mathrm{~g}, 5 \mathrm{mmol})$, thiourea $(1.52 \mathrm{~g}, 20 \mathrm{mmol})$ dissolved in tertahydrofuran $(5 \mathrm{~mL})$ was taken. The contents were heated at $120-150^{\circ} \mathrm{C}$ in an oil bath for 24 $30 \mathrm{~h}$. After the reaction was completed, it was extracted with dichloromethane. The organic layer was washed with water, brine solution and dried over anhydrous $\mathrm{Na}_{2} \mathrm{SO}_{4}$. The resultant solid was recrystallized from methanol to get 10a. Yield $1.42 \mathrm{~g}(72 \%)$; white solid; m. p. $134-136{ }^{\circ} \mathrm{C} ;{ }^{1} \mathrm{H}$ NMR (300 MHz, DMSO-d $): \delta=4.21\left(\mathrm{~s}, 4 \mathrm{H}, \mathrm{CH}_{2}\right), 7.23-7.56(\mathrm{~m}, 10 \mathrm{H}, \mathrm{Ar}-\mathrm{H}) ;{ }^{13} \mathrm{C}$ NMR $(300$ $\left.\mathrm{MHz}, \mathrm{DMSO}-d_{6}\right): \delta=55.4\left(\mathrm{CH}_{2}\right), 161.1(\mathrm{C}-2 \& \mathrm{C}-5), 125.9,128.6,129.2,133.4$ (aromatic carbons); IR (KBr): $v=1631(\mathrm{C}=\mathrm{N}), 1345,1128\left(\mathrm{SO}_{2}\right)$; Anal. Calcd. for $\mathrm{C}_{16} \mathrm{H}_{14} \mathrm{~N}_{2} \mathrm{O}_{4} \mathrm{~S}_{3}(394.49)$ : C, 48.71; H, 3.58; N, 7.10. Found: C, 48.77; H, 3.62; N, 7.07.

2,5-Bis(4-methylphenylsulfonylmethyl)-1,3,4-thiadiazole (10b). Yield $1.44 \mathrm{~g}$ (68\%); white solid; m. p. $156-158{ }^{\circ} \mathrm{C}$; ${ }^{1} \mathrm{H}$ NMR (300 MHz, DMSO- $\left.d_{6}\right): \delta=2.27$ (s, 6H, Ar-CH $), 4.18$ (s, 4H, $\left.\mathrm{CH}_{2}\right), 7.25-7.59(\mathrm{~m}, 8 \mathrm{H}, \mathrm{Ar}-\mathrm{H}) ;{ }^{13} \mathrm{C} \mathrm{NMR}\left(300 \mathrm{MHz}, \mathrm{DMSO}-d_{6}\right): \delta=21.9\left(\mathrm{Ar}_{-} \mathrm{CH}_{3}\right), 54.7$ $\left(\mathrm{CH}_{2}\right), 161.5$ (C-2 \& C-5), 127.4, 128.9, 131.3, 134.6 (aromatic carbons); IR (KBr): $v=1632$ $(\mathrm{C}=\mathrm{N}), 1342,1145\left(\mathrm{SO}_{2}\right)$; Anal. Calcd. for $\mathrm{C}_{18} \mathrm{H}_{18} \mathrm{~N}_{2} \mathrm{O}_{4} \mathrm{~S}_{3}$ (422.54): C, 51.16; H, 4.29; N, 6.63. Found: C, 51.12; H, 4.25; N, 6.67.

2,5-Bis(4-chlorophenylsulfonylmethyl)-1,3,4-thiadiazole (10c). Yield $1.73 \mathrm{~g}$ (75\%); white solid; m. p. $173-175{ }^{\circ} \mathrm{C}$; ${ }^{1} \mathrm{H}$ NMR (300 MHz, DMSO-d $): \delta=4.24$ (s, 4H, $\left.\mathrm{CH}_{2}\right), 7.38-7.81$ (m, $8 \mathrm{H}, \mathrm{Ar}-\mathrm{H}) ;{ }^{13} \mathrm{C}$ NMR $\left(300 \mathrm{MHz}, \mathrm{DMSO}-d_{6}\right): \delta=55.6\left(\mathrm{CH}_{2}\right), 160.8(\mathrm{C}-2 \& \mathrm{C}-5), 126.4,129.1$, 132.8, 136.5 (aromatic carbons); IR $(\mathrm{KBr}): v=1627(\mathrm{C}=\mathrm{N}), 1348,1136\left(\mathrm{SO}_{2}\right)$; Anal. Calcd. for $\mathrm{C}_{16} \mathrm{H}_{12} \mathrm{Cl}_{2} \mathrm{~N}_{2} \mathrm{O}_{4} \mathrm{~S}_{3}$ (463.38): C, 41.47; H, 2.61; N, 6.05. Found: C, 41.52; H, 2.62; N, 6.11 .

\section{2,5-Bis(benzylsulfonylmethyl)-1,3,4-thiadiazole (11a). Typical procedure}

In a sealed test tube, a mixture of 8a $(2.03 \mathrm{~g}, 5 \mathrm{mmol})$, thiourea $(1.52 \mathrm{~g}, 20 \mathrm{mmol})$ dissolved in tetrahydrofuran $(5 \mathrm{~mL})$ was taken. The contents were heated at $120-150^{\circ} \mathrm{C}$ in an oil bath for 20 $22 \mathrm{~h}$. After the reaction was completed, it was extracted with dichloromethane. The organic layer was washed with water, brine solution and dried over anhydrous $\mathrm{Na}_{2} \mathrm{SO}_{4}$. The resultant solid was recrystallized from methanol to get 11a. Yield $1.52 \mathrm{~g}(72 \%)$; white solid; m. p. $210-212{ }^{\circ} \mathrm{C} ;{ }^{1} \mathrm{H}$ 
NMR (300 MHz, $\left.\mathrm{CDCl}_{3}+\mathrm{DMSO}-d_{6}\right): \delta=4.12\left(\mathrm{~s}, 4 \mathrm{H}, \mathrm{CH}_{2}\right), 4.65\left(\mathrm{~s}, 4 \mathrm{H}, \mathrm{Ar}-\mathrm{CH}_{2}\right), 7.23-7.41(\mathrm{~m}$, $10 \mathrm{H}, \mathrm{Ar}-\mathrm{H}) ;{ }^{13} \mathrm{C} \mathrm{NMR}\left(300 \mathrm{MHz}, \mathrm{CDCl}_{3}+\mathrm{DMSO}-d_{6}\right): \delta=56.1\left(\mathrm{CH}_{2}\right), 58.0\left(\mathrm{Ar}-\mathrm{CH}_{2}\right), 161.8(\mathrm{C}-$ 2 \& C-5), 126.1, 129.3, 132.8, 134.4 (aromatic carbons); IR (KBr): $v=1633(\mathrm{C}=\mathrm{N}), 1332,1125$ $\left(\mathrm{SO}_{2}\right)$; Anal. Calcd. for $\mathrm{C}_{18} \mathrm{H}_{18} \mathrm{~N}_{2} \mathrm{O}_{4} \mathrm{~S}_{3}$ (422.54): C, 51.16; H, 4.29; N, 6.63. Found: C, 51.18; H, $4.33 ; \mathrm{N}, 6.67$.

2,5-Bis(4-methylbenzylsulfonylmethyl)-1,3,4-thiadiazole (11b). Yield $1.55 \mathrm{~g}$ (69\%); white crystals; m. p. $238-240{ }^{\circ} \mathrm{C} ;{ }^{1} \mathrm{H}$ NMR $\left(300 \mathrm{MHz}, \mathrm{CDCl}_{3}+\mathrm{DMSO}-d_{6}\right): \delta=2.25\left(\mathrm{~s}, 6 \mathrm{H}, \mathrm{Ar}_{-} \mathrm{CH}_{3}\right)$, $4.09\left(\mathrm{~s}, 4 \mathrm{H}, \mathrm{CH}_{2}\right), 4.64\left(\mathrm{~s}, 4 \mathrm{H}, \mathrm{Ar}-\mathrm{CH}_{2}\right), 7.16-7.44(\mathrm{~m}, 8 \mathrm{H}, \mathrm{Ar}-\mathrm{H}) ;{ }^{13} \mathrm{C} \mathrm{NMR}(300 \mathrm{MHz}$, $\left.\mathrm{CDCl}_{3}+\mathrm{DMSO}-d_{6}\right): \delta=21.4\left(\mathrm{Ar}-\mathrm{CH}_{3}\right), 55.9\left(\mathrm{CH}_{2}\right), 58.2\left(\mathrm{Ar}-\mathrm{CH}_{2}\right), 162.4(\mathrm{C}-2$ \& C-5), 126.4, 129.3, 131.3, 133.6 (aromatic carbons); IR (KBr): $v=1625(\mathrm{C}=\mathrm{N}), 1338,1143\left(\mathrm{SO}_{2}\right)$; Anal. Calcd. for $\mathrm{C}_{20} \mathrm{H}_{22} \mathrm{~N}_{2} \mathrm{O}_{4} \mathrm{~S}_{3}$ (450.59): C, 53.31; H, 4.92; N, 6.22. Found: C, 53.37; H, 4.96; N, 6.18. 2,5-Bis(4-chlorobenzylsulfonylmethyl)-1,3,4-thiadiazole (11c). Yield $1.89 \mathrm{~g}$ (77\%); white solid; m. p. 253-255 ${ }^{\circ} \mathrm{C} ;{ }^{1} \mathrm{H}$ NMR (300 MHz, DMSO- $\left.d_{6}\right): \delta=4.14$ (s, 4H, $\left.\mathrm{CH}_{2}\right), 4.69$ (s, 4H, Ar$\left.\mathrm{CH}_{2}\right), 7.34-7.62(\mathrm{~m}, 8 \mathrm{H}, \mathrm{Ar}-\mathrm{H}) ;{ }^{13} \mathrm{C}$ NMR $\left(300 \mathrm{MHz}, \mathrm{DMSO}-d_{6}\right): \delta=56.3\left(\mathrm{CH}_{2}\right), 57.9(\mathrm{Ar}-$ $\mathrm{CH}_{2}$ ), 162.4 (C-2 \& C-5), 126.6, 129.5, 133.9, 137.5 (aromatic carbons); IR (KBr): $v=1628$ $(\mathrm{C}=\mathrm{N})$, 1334, $1138\left(\mathrm{SO}_{2}\right)$; Anal. Calcd. for $\mathrm{C}_{18} \mathrm{H}_{16} \mathrm{Cl}_{2} \mathrm{~N}_{2} \mathrm{O}_{4} \mathrm{~S}_{3}$ (491.43): C, 43.99; H, 3.28; N, 5.70. Found: C, 43.92; H, 3.25; N, 5.66.

2-(Benzylsulfonylmethyl)-5-(phenylsulfonylmethyl)-1,3,4-thiadiazole $\quad$ (12a). Typical procedure

In a sealed test tube, a mixture of 9a $(1.96 \mathrm{~g}, 5 \mathrm{mmol})$, thiourea $(1.52 \mathrm{~g}, 20 \mathrm{mmol})$ dissolved in tetrahydrofuran $(5 \mathrm{~mL})$ was taken. The contents were heated at $120-150^{\circ} \mathrm{C}$ in an oil bath for $22-$ $26 \mathrm{~h}$. After the reaction was completed, it was extracted with dichloromethane. The organic layer was washed with water, brine solution and dried over anhydrous $\mathrm{Na}_{2} \mathrm{SO}_{4}$. The resultant solid was recrystallized from methanol to get 12a. Yield $1.49 \mathrm{~g}(73 \%)$; white solid; m. p. 160-162 ${ }^{\circ} \mathrm{C} ;{ }^{1} \mathrm{H}$ NMR (300 MHz, DMSO- $\left.d_{6}\right): \delta=3.93\left(\mathrm{~s}, 2 \mathrm{H}, \mathrm{SO}_{2}-\mathrm{CH}_{2}\right), 4.25$ (s, 2H, Ar-CH $), 4.52$ (s, 2H, $\left.\mathrm{ArSO}_{2}-\mathrm{CH}_{2}\right), 7.28-7.62(\mathrm{~m}, 10 \mathrm{H}, \mathrm{Ar}-\mathrm{H}) ;{ }^{13} \mathrm{C}$ NMR (300 MHz, DMSO- $\left.d_{6}\right): \delta=52.9\left(\mathrm{SO}_{2}-\mathrm{CH}_{2}\right)$, $57.3\left(\mathrm{Ar}^{-} \mathrm{CH}_{2}\right), 59.0\left(\mathrm{ArSO}_{2}-\mathrm{CH}_{2}\right), 156.8$ (C-2), $159.2(\mathrm{C}-5), 125.2,126.4,127.3,129.5,131.8$, 131.9, 133.5, 135.3 (aromatic carbons); IR (KBr): $v=1621(\mathrm{C}=\mathrm{N}), 1320,1144\left(\mathrm{SO}_{2}\right)$; Anal. Calcd. for $\mathrm{C}_{17} \mathrm{H}_{16} \mathrm{~N}_{2} \mathrm{O}_{4} \mathrm{~S}_{3}$ (408.51): C, 49.98; H, 3.95; N, 6.86. Found: C, 50.05; H, 3.98; N, 6.90.

2-(4-Methylbenzylsulfonylmethyl)-5-(4-methylphenylsulfonylmethyl)-1,3,4-thiadiazole

(12b). Yield 1.70 g (78\%); white solid; m. p. 182-184 ${ }^{\circ} \mathrm{C} ;{ }^{1} \mathrm{H}$ NMR (300 MHz, DMSO- $\left.d_{6}\right): \delta=$ $2.22 \& 2.26\left(\mathrm{~s}, 6 \mathrm{H}, \mathrm{Ar}-\mathrm{CH}_{3}\right), 3.91\left(\mathrm{~s}, 2 \mathrm{H}, \mathrm{SO}_{2}-\mathrm{CH}_{2}\right), 4.22\left(\mathrm{~s}, 2 \mathrm{H}, \mathrm{Ar}-\mathrm{CH}_{2}\right), 4.49\left(\mathrm{~s}, 2 \mathrm{H}, \mathrm{ArSO}_{2}-\right.$ $\left.\mathrm{CH}_{2}\right), 7.22-7.58(\mathrm{~m}, 8 \mathrm{H}, \mathrm{Ar}-\mathrm{H}) ;{ }^{13} \mathrm{C} \mathrm{NMR}\left(300 \mathrm{MHz}, \mathrm{DMSO}-d_{6}\right): \delta=21.2 \& 21.6\left(\mathrm{Ar}-\mathrm{CH}_{3}\right)$, $53.4\left(\mathrm{SO}_{2}-\mathrm{CH}_{2}\right), 57.8\left(\mathrm{Ar}-\mathrm{CH}_{2}\right), 59.3\left(\mathrm{ArSO}_{2}-\mathrm{CH}_{2}\right), 157.0(\mathrm{C}-2), 158.8(\mathrm{C}-5), 126.3,127.5$, 128.7, 129.4, 129.9, 131.8, 134.2, 135.7 (aromatic carbons); IR $(\mathrm{KBr}): v=1636(\mathrm{C}=\mathrm{N}), 1322$, $1140\left(\mathrm{SO}_{2}\right)$; Anal. Calcd. for $\mathrm{C}_{19} \mathrm{H}_{20} \mathrm{~N}_{2} \mathrm{O}_{4} \mathrm{~S}_{3}$ (436.57): C, 52.27; H, 4.62; N, 6.42. Found: C, 52.24; H, 4.60; N, 6.45.

2-(4-Chlorobenzylsulfonylmethyl)-5-(4-chlorophenylsulfonylmethyl)-1,3,4-thiadiazole (12c). Yield 1.79 g (75\%); white crystals; m. p. $201-203{ }^{\circ} \mathrm{C} ;{ }^{1} \mathrm{H}$ NMR (300 MHz, DMSO- $\left.d_{6}\right): \delta=3.94$ 
(s, 2H, $\left.\mathrm{SO}_{2}-\mathrm{CH}_{2}\right), 4.20$ (s, 2H, Ar- $\left.\mathrm{CH}_{2}\right), 4.54\left(\mathrm{~s}, 2 \mathrm{H}, \mathrm{ArSO}_{2}-\mathrm{CH}_{2}\right), 7.37-7.68(\mathrm{~m}, 8 \mathrm{H}, \mathrm{Ar}-\mathrm{H}) ;{ }^{13} \mathrm{C}$ NMR (300 MHz, DMSO- $\left.d_{6}\right): \delta=53.1\left(\mathrm{SO}_{2}-\mathrm{CH}_{2}\right), 57.5\left(\mathrm{Ar}-\mathrm{CH}_{2}\right), 58.8\left(\mathrm{ArSO}_{2}-\mathrm{CH}_{2}\right), 157.4(\mathrm{C}-$ 2), 160.2 (C-5), 125.2, 126.4, 127.3, 129.5, 131.8, 131.9, 133.5, 138.3 (aromatic carbons); IR $(\mathrm{KBr}): v=1630(\mathrm{C}=\mathrm{N}), 1335,1138\left(\mathrm{SO}_{2}\right)$; Anal. Calcd. for $\mathrm{C}_{17} \mathrm{H}_{14} \mathrm{Cl}_{2} \mathrm{~N}_{2} \mathrm{O}_{4} \mathrm{~S}_{3}$ (477.41): C, 42.77; H, 2.96; N, 5.87. Found: C, 42.82; H, 2.99; N, 5.83.

\section{3,5-Bis(phenylsulfonylmethyl)-4-amino-1,2,4-triazoles (13a). Typical procedure}

To a solution of $7 \mathbf{a}(1.89 \mathrm{~g}, 5 \mathrm{mmol})$ in $n$-butanol $(25 \mathrm{~mL})$, hydrazine hydrate $(0.75 \mathrm{~g}, 15 \mathrm{mmol})$ was added and refluxed for 3-4 h. Then, $\mathrm{KOH}(0.56 \mathrm{~g}, 10 \mathrm{mmol})$ was added to the reaction media and the precipitate formed was filtered. The solid obtained was acidified with conc. $\mathrm{HCl}$ to $\mathrm{pH} \approx 3$ and washed with water. The resultant solid was recrystallized from ethanol to get 13a. Yield 1.39 g (71\%); yellow solid; m. p. $139-141{ }^{\circ} \mathrm{C} ;{ }^{1} \mathrm{H}$ NMR $\left(300 \mathrm{MHz}, \mathrm{DMSO}-d_{6}\right): \delta=4.22$ $\left(\mathrm{s}, 4 \mathrm{H}, \mathrm{CH}_{2}\right), 5.57$ (bs, 2H, NH $\left.\mathrm{N}_{2}\right), 7.16-7.57$ (m, 10H, Ar-H); ${ }^{13} \mathrm{C}$ NMR (300 MHz, DMSO- $\left.d_{6}\right): \delta$ $=55.6\left(\mathrm{CH}_{2}\right), 161.7(\mathrm{C}-3 \& \mathrm{C}-5), 126.4,129.5,132.1,133.7$ (aromatic carbons); IR $(\mathrm{KBr}): v=$ 3257, $3238\left(\mathrm{NH}_{2}\right), 1630(\mathrm{C}=\mathrm{N}), 1338,1135\left(\mathrm{SO}_{2}\right)$; Anal. Calcd. for $\mathrm{C}_{16} \mathrm{H}_{16} \mathrm{~N}_{4} \mathrm{O}_{4} \mathrm{~S}_{2}$ (392.45): C, 48.97; H, 4.11; N, 14.28. Found: C, 49.05; H, 4.14; N, 14.35.

3,5-Bis(4-methylphenylsulfonylmethyl)-4-amino-1,2,4-triazoles (13b). Yield $1.59 \mathrm{~g}$ (76\%); yellow solid; m. p. $162-164{ }^{\circ} \mathrm{C} ;{ }^{1} \mathrm{H}$ NMR $\left(300 \mathrm{MHz}, \mathrm{DMSO}-d_{6}\right): \delta=2.23\left(\mathrm{~s}, 6 \mathrm{H}, \mathrm{Ar}-\mathrm{CH}_{3}\right), 4.19$ (s, 4H, $\left.\mathrm{CH}_{2}\right), 5.63$ (bs, 2H, NH ), 7.18-7.62 (m, 8H, Ar-H); ${ }^{13} \mathrm{C}$ NMR (300 MHz, DMSO- $\left.d_{6}\right): \delta=$ $21.5\left(\mathrm{Ar}-\mathrm{CH}_{3}\right), 54.6\left(\mathrm{CH}_{2}\right), 161.8(\mathrm{C}-3 \& \mathrm{C}-5), 125.3,127.5,129.1,132.4$ (aromatic carbons); IR $(\mathrm{KBr}): v=3253,3232\left(\mathrm{NH}_{2}\right), 1638(\mathrm{C}=\mathrm{N}), 1330,1132\left(\mathrm{SO}_{2}\right)$; Anal. Calcd. for $\mathrm{C}_{18} \mathrm{H}_{20} \mathrm{~N}_{4} \mathrm{O}_{4} \mathrm{~S}_{2}$ (420.51): C, 51.41; H, 4.79; N, 13.32. Found: C, 51.47; H, 4.75; N, 13.38 .

3,5-Bis(4-chlorophenylsulfonylmethyl)-4-amino-1,2,4-triazoles (13c). Yield: $1.68 \mathrm{~g}$ (73\%); yellow solid; m. p. $171-173{ }^{\circ} \mathrm{C}$; ${ }^{1} \mathrm{H}$ NMR (300 MHz, DMSO- $\left.d_{6}\right): \delta=4.16\left(\mathrm{~s}, 4 \mathrm{H}, \mathrm{CH}_{2}\right), 5.58(\mathrm{bs}$, $\left.2 \mathrm{H}, \mathrm{NH}_{2}\right), 7.34-7.78(\mathrm{~m}, 8 \mathrm{H}, \mathrm{Ar}-\mathrm{H}) ;{ }^{13} \mathrm{C} \mathrm{NMR}\left(300 \mathrm{MHz}, \mathrm{DMSO}-d_{6}\right): \delta=55.3\left(\mathrm{CH}_{2}\right), 162.5(\mathrm{C}-$ 3 \& C-5), 125.9, 130.7, 133.2, 136.8 (aromatic carbons); IR (KBr): $v=3250,3236\left(\mathrm{NH}_{2}\right), 1634$ $(\mathrm{C}=\mathrm{N}), 1336,1128\left(\mathrm{SO}_{2}\right)$; Anal. Calcd. for $\mathrm{C}_{16} \mathrm{H}_{14} \mathrm{Cl}_{2} \mathrm{~N}_{4} \mathrm{O}_{4} \mathrm{~S}_{2}$ (461.34): C, 41.65; H, 3.06; N, 12.14. Found: C, 41.61; H, 3.08; N, 12.18 .

\section{3,5-Bis(benzylsulfonylmethyl)-4-amino-1,2,4-triazoles (14a). Typical procedure}

To a solution of $\mathbf{8 a}(2.03 \mathrm{~g}, 5 \mathrm{mmol})$ in $25 \mathrm{~mL} n$-butanol, hydrazine hydrate $(0.75 \mathrm{~g}, 15 \mathrm{mmol})$ was added and refluxed for 5-6 $\mathrm{h}$. Then $\mathrm{KOH}(0.56 \mathrm{~g}, 10 \mathrm{mmol})$ was added to the reaction media and the precipitate formed was filtered. The solid obtained was acidified with conc. $\mathrm{HCl}$ to $\mathrm{pH} \approx$ 3 and washed with water. The resultant solid was recrystallized from ethanol to get 14a. Yield 1.40 g (67\%); yellow solid; m. p. $215-217{ }^{\circ} \mathrm{C}$; ${ }^{1} \mathrm{H}$ NMR $\left(300 \mathrm{MHz}, \mathrm{DMSO}-d_{6}\right): \delta=4.14$ (s, $4 \mathrm{H}$, $\left.\mathrm{CH}_{2}\right), 4.64$ (s, 4H, Ar- $\left.\mathrm{CH}_{2}\right), 5.66$ (bs, $\left.2 \mathrm{H}, \mathrm{NH}_{2}\right), 7.27-7.45(\mathrm{~m}, 10 \mathrm{H}, \mathrm{Ar}-\mathrm{H}) ;{ }^{13} \mathrm{C} \mathrm{NMR}(300 \mathrm{MHz}$, DMSO- $\left.d_{6}\right): \delta=55.7\left(\mathrm{CH}_{2}\right), 58.7\left(\mathrm{Ar}-\mathrm{CH}_{2}\right), 163.2(\mathrm{C}-3$ \& C-5), 128.9, 129.6, 131.3, 133.5 (aromatic carbons); IR (KBr): $v=3251,3242\left(\mathrm{NH}_{2}\right), 1623(\mathrm{C}=\mathrm{N}), 1334,1128\left(\mathrm{SO}_{2}\right)$; Anal. Calcd. for $\mathrm{C}_{18} \mathrm{H}_{20} \mathrm{~N}_{4} \mathrm{O}_{4} \mathrm{~S}_{2}$ (420.51): C, 51.41; H, 4.79; N, 13.32. Found: C, 51.45; H, 4.76; N, 13.35 . 
3,5-Bis(4-methylbenzylsulfonylmethyl)-4-amino-1,2,4-triazoles (14b). Yield $1.65 \mathrm{~g}$ (74\%); yellow solid; m. p. $235-237{ }^{\circ} \mathrm{C}$; ${ }^{1} \mathrm{H}$ NMR (300 MHz, DMSO- $\left.d_{6}\right): \delta=4.11\left(\mathrm{~s}, 4 \mathrm{H}, \mathrm{CH}_{2}\right), 4.59$ (s, $\left.4 \mathrm{H}, \mathrm{Ar}-\mathrm{CH}_{2}\right), 5.62$ (bs, 2H, $\left.\mathrm{NH}_{2}\right), 7.16-7.42(\mathrm{~m}, 8 \mathrm{H}, \mathrm{Ar}-\mathrm{H}) ;{ }^{13} \mathrm{C}$ NMR (300 MHz, DMSO- $\left.d_{6}\right): \delta$ $=21.8\left(\mathrm{Ar}-\mathrm{CH}_{3}\right), 55.4\left(\mathrm{CH}_{2}\right), 58.4\left(\mathrm{Ar}-\mathrm{CH}_{2}\right), 162.8(\mathrm{C}-3$ \& $\mathrm{C}-5), 126.1,128.7,131.7,134.2$ (aromatic carbons); IR (KBr): $v=3258,3244\left(\mathrm{NH}_{2}\right), 1632(\mathrm{C}=\mathrm{N}), 1330,1132\left(\mathrm{SO}_{2}\right)$; Anal. Calcd. for $\mathrm{C}_{20} \mathrm{H}_{24} \mathrm{~N}_{4} \mathrm{O}_{4} \mathrm{~S}_{2}$ (448.56): C, 53.55; H, 5.39; N, 12.49. Found: C, 53.50; H, 5.43; N, 12.55 .

3,5-Bis(4-chlorobenzylsulfonylmethyl)-4-amino-1,2,4-triazoles (14c). Yield $1.76 \mathrm{~g}$ (72\%); yellow crystals; m. p. $258-260{ }^{\circ} \mathrm{C} ;{ }^{1} \mathrm{H}$ NMR (300 MHz, DMSO- $\left.d_{6}\right): \delta=4.17\left(\mathrm{~s}, 4 \mathrm{H}, \mathrm{CH}_{2}\right), 4.62$ $\left(\mathrm{s}, 4 \mathrm{H}, \mathrm{Ar}-\mathrm{CH}_{2}\right), 5.68$ (bs, $\left.2 \mathrm{H}, \mathrm{NH}_{2}\right), 7.31-7.65(\mathrm{~m}, 8 \mathrm{H}, \mathrm{Ar}-\mathrm{H}) ;{ }^{13} \mathrm{C}$ NMR $\left(300 \mathrm{MHz}, \mathrm{DMSO}-d_{6}\right)$ : $\delta=54.9\left(\mathrm{CH}_{2}\right), 56.7\left(\mathrm{Ar}-\mathrm{CH}_{2}\right), 163.2(\mathrm{C}-3$ \& C-5), 126.4, 129.5, 131.3, 135.5 (aromatic carbons); IR $(\mathrm{KBr}): v=3262,3249\left(\mathrm{NH}_{2}\right), 1628(\mathrm{C}=\mathrm{N}), 1325,1141\left(\mathrm{SO}_{2}\right)$; Anal. Calcd. for $\mathrm{C}_{18} \mathrm{H}_{18} \mathrm{Cl}_{2} \mathrm{~N}_{4} \mathrm{O}_{4} \mathrm{~S}_{2}$ (489.40): C, 44.18; H, 3.71; N, 11.45. Found: C, 44.42; H, 3.70; N, 11.50 .

\section{3-(Benzylsulfonylmethyl)-5-(phenylsulfonylmethyl)-4-amino-1,2,4-triazoles (15a). Typical procedure}

To a solution of 9a $(1.96 \mathrm{~g}, 5 \mathrm{mmol})$ in $n$-butanol $(25 \mathrm{~mL})$, hydrazine hydrate $(0.75 \mathrm{~g}, 15 \mathrm{mmol})$ was added and refluxed for $4-5 \mathrm{~h}$. To this $\mathrm{KOH}(0.56 \mathrm{~g}, 10 \mathrm{mmol})$ was added and the precipitate formed was filtered. The solid obtained was acidified with conc. $\mathrm{HCl}$ to $\mathrm{pH} \approx 3$ and washed with water. The resultant solid was recrystallized from ethanol to get 15a. Yield $1.38 \mathrm{~g}(68 \%)$; yellow solid; m. p. 166-168 ${ }^{\circ} \mathrm{C}$; ${ }^{1} \mathrm{H}$ NMR (300 MHz, $\left.\mathrm{CDCl}_{3}+\mathrm{DMSO}-d_{6}\right): \delta=3.92\left(\mathrm{~s}, 2 \mathrm{H}, \mathrm{SO}_{2}-\mathrm{CH}_{2}\right.$ ), $4.21\left(\mathrm{~s}, 2 \mathrm{H}, \mathrm{Ar}-\mathrm{CH}_{2}\right), 4.50$ (s, 2H, $\mathrm{ArSO}_{2}-\mathrm{CH}_{2}$ ), 5.60 (bs, 2H, NH ), 7.29-7.47 (m, 10H, Ar-H); ${ }^{13} \mathrm{C} \mathrm{NMR}\left(300 \mathrm{MHz}, \mathrm{CDCl}_{3}+\mathrm{DMSO}-d_{6}\right): \delta=53.2\left(\mathrm{SO}_{2}-\mathrm{CH}_{2}\right), 56.9\left(\mathrm{Ar}_{-} \mathrm{CH}_{2}\right), 59.1\left(\mathrm{ArSO}_{2}-\right.$ $\mathrm{CH}_{2}$ ), 159.9 (C-3), 161.4 (C-5), 125.3, 125.9, 126.7, 127.4, 129.1, 131.3, 132.8, 134.3 (aromatic carbons); IR $(\mathrm{KBr}): v=3259,3241\left(\mathrm{NH}_{2}\right), 1633(\mathrm{C}=\mathrm{N}), 1344,1136\left(\mathrm{SO}_{2}\right)$; Anal. Calcd. for $\mathrm{C}_{17} \mathrm{H}_{18} \mathrm{~N}_{4} \mathrm{O}_{4} \mathrm{~S}_{2}$ (406.48): C, 50.23; H, 4.46; N, 13.78. Found: C, 50.27; H, 4.42; N, 13.85 .

3-(4-Methylbenzylsulfonylmethyl)-5-(4-methylphenylsulfonylmethyl)-4-amino-1,2,4-

triazoles (15b). Yield $1.67 \mathrm{~g}$ (77\%); yellow solid; m. p. 178-180 ${ }^{\circ} \mathrm{C}$; ${ }^{1} \mathrm{H}$ NMR (300 $\mathrm{MHz}$, $\left.\mathrm{CDCl}_{3}+\mathrm{DMSO}-d_{6}\right): \delta=2.21 \& 2.25\left(\mathrm{~s}, 6 \mathrm{H}, \mathrm{Ar}-\mathrm{CH}_{3}\right), 3.90\left(\mathrm{~s}, 2 \mathrm{H}, \mathrm{SO}_{2}-\mathrm{CH}_{2}\right), 4.18$ (s, 2H, Ar$\left.\mathrm{CH}_{2}\right), 4.48\left(\mathrm{~s}, 2 \mathrm{H}, \mathrm{ArSO}_{2}-\mathrm{CH}_{2}\right), 5.58\left(\mathrm{bs}, 2 \mathrm{H}, \mathrm{NH}_{2}\right), 7.26-7.52(\mathrm{~m}, 8 \mathrm{H}, \mathrm{Ar}-\mathrm{H}) ;{ }^{13} \mathrm{C}$ NMR $(300$ $\left.\mathrm{MHz}, \mathrm{CDCl}_{3}+\mathrm{DMSO}-d_{6}\right): \delta=21.2 \& 21.7\left(\mathrm{Ar}-\mathrm{CH}_{3}\right), 53.7\left(\mathrm{SO}_{2}-\mathrm{CH}_{2}\right), 57.5\left(\mathrm{Ar}-\mathrm{CH}_{2}\right), 59.4$ $\left(\mathrm{ArSO}_{2}-\mathrm{CH}_{2}\right), 158.7$ (C-3), 161.7 (C-5), 122.1, 125.5, 127.1, 129.5, 131.4, 132.4, 133.0, 135.8 (aromatic carbons); IR $(\mathrm{KBr}): v=3256,3234\left(\mathrm{NH}_{2}\right), 1625(\mathrm{C}=\mathrm{N}), 1343,1142\left(\mathrm{SO}_{2}\right)$; Anal. Calcd. for $\mathrm{C}_{19} \mathrm{H}_{22} \mathrm{~N}_{4} \mathrm{O}_{4} \mathrm{~S}_{2}$ (434.53): C, 52.52; H, 5.10; N, 12.89. Found: C, 52.60; H, 5.13; N, 12.93 .

\section{3-(4-Chlorobenzylsulfonylmethyl)-5-(4-chlorophenylsulfonylmethyl)-4-amino-1,2,4-}

triazoles (15c). Yield $1.66 \mathrm{~g}$ (70\%); yellow solid; m. p. 207-209 ${ }^{\circ} \mathrm{C}$; ${ }^{1} \mathrm{H}$ NMR (300 $\mathrm{MHz}$, $\left.\mathrm{CDCl}_{3}+\mathrm{DMSO}-d_{6}\right): \delta=3.94\left(\mathrm{~s}, 2 \mathrm{H}, \mathrm{SO}_{2}-\mathrm{CH}_{2}\right), 4.23\left(\mathrm{~s}, 2 \mathrm{H}, \mathrm{Ar}-\mathrm{CH}_{2}\right), 4.53\left(\mathrm{~s}, 2 \mathrm{H}, \mathrm{ArSO}_{2}-\mathrm{CH}_{2}\right)$, 5.64 (bs, $\left.2 \mathrm{H}, \mathrm{NH}_{2}\right), 7.37-7.70(\mathrm{~m}, 8 \mathrm{H}, \mathrm{Ar}-\mathrm{H}) ;{ }^{13} \mathrm{C} \mathrm{NMR}\left(300 \mathrm{MHz}, \mathrm{CDCl}_{3}+\mathrm{DMSO}-d_{6}\right): \delta=53.5$ $\left(\mathrm{SO}_{2}-\mathrm{CH}_{2}\right), 57.3\left(\mathrm{Ar}-\mathrm{CH}_{2}\right), 58.7\left(\mathrm{ArSO}_{2}-\mathrm{CH}_{2}\right), 159.3(\mathrm{C}-3), 161.3$ (C-5), 126.9, 127.0, 127.7, 
129.3, 131.3, 132.8, 135.4, 137.9 (aromatic carbons); IR (KBr): $v=3260,3245\left(\mathrm{NH}_{2}\right), 1629$ $(\mathrm{C}=\mathrm{N}), 1352,1139\left(\mathrm{SO}_{2}\right)$; Anal. Calcd. for $\mathrm{C}_{17} \mathrm{H}_{16} \mathrm{Cl}_{2} \mathrm{~N}_{4} \mathrm{O}_{4} \mathrm{~S}_{2}$ (475.37): $\mathrm{C}, 42.95 ; \mathrm{H}, 3.39 ; \mathrm{N}$, 11.79. Found: C, 43.01; H, 3.40; N, 11.73 .

\section{Acknowledgements}

The authors are thankful to Department of Science and Technology (DST) New Delhi, India for the financial assistance under major research project.

\section{References}

1. Omar, F. A.; Mahfouz, N. M.; Rahman, M. A. Eur. J. Med. Chem. 1996, 31, 819.

2. (a) Goswami, B. N.; Kataky, J. C. S.; Baruah, J. N.; Nath, S. C. J. Heterocycl. Chem. 1984, 21, 205. (b) Holla, B. S.; Poojary, K. N.; Kalluraya, B.; Gowda, P. V. Indian J. Heterocycl. Chem. 1996, 5, 273.

3. Omar, M. T. Arch. Pharm. Res. (Seoul) 1997, 20, 602.

4. (a) Hamad, M. M.; Said, S. A.; El-Ekyabi, Y. M. Monatsh. Chem. 1996, 127, 549. (b) Matsumoto, K.; Kuwamura, Y.; Yasuda, Y.; Tanimoto, T.; Matsumoto, K.; Yoshida, T.; Shoji, J. J. Antibiot. (Tokyo) 1998, 42, 1465. (c) Papakonstantinou, G. S.; Marakos, P.; Tsantili, K. A.; Chytyroglon, L. A. Pharmazie 1998, 53, 300.

5. Tandon, V. K.; Chhor, R. B. Synth. Commun. 2001, 31, 1727.

6. Diana, G. D.; Volkots, D. L.; Nitz, T. J.; Biailly, T. R.; Long, M. A.; Vesico, N.; Aldous, A.; Pevear, D. C.; Dukto, F. J. J. Med. Chem. 1994, 37, 2421.

7. Liras, S.; Allen, M. P.; Segelstein, B. E. Synth. Commun. 2000, 30, 437.

8. Carlsen, H. J.; Jorgensen, K. B. J. Heterocycl. Chem. 1994, $31,805$.

9. Tully, W. R.; Cardner, C. R.; Gillespie, R. J.; Westwood, R. J. Med. Chem. 1991, 34, 2060.

10. (a) Al-Talib, M.; Tashtoush, H.; Odeh, N. Synth. Commun. 1990, 20, 1811. (b) Kerr, N. V.; Ott, D. G.; Hayes, F. N. J. Am. Chem. Soc. 1960, 82, 186.

11. Theocharis, A. B.; Alexandrou, N. E. J. Heterocycl. Chem. 1990, 27, 1685.

12. Short, F. W.; Long, L. M. J. Heterocycl. Chem. 1969, 6, 707.

13. Bentiss, F.; Lagrenee, M. J. Heterocycl. Chem. 1999, 36, 1029.

14. (a) Hill, D. L. Cancer Chemother. Pharmacol. 1980, 4, 215. (b) Asbury, R. F.; Kramar, A.; Haller, D. G. Am. J. Clin. Oncol. 1987, 10, 380.

15. Dogan, H. N.; Duran, A.; Rollas, S.; Sener, G.; Uysal, M. K.; Gulen, D. Bioorg. Med. Chem. 2002, 10, 2893.

16. Rollas, S.; Karakus, S.; Durgun, B. B.; Kiraz, M.; Erdeniz, H. Farmaco 1996, 51, 811. 
17. Song, Y.; Connor, T.; Sercel, A. D.; Sorenson, R. J.; Doubleday, R.; Unangst, P. C.; Roth, B. D.; Beylin, V. G.; Gilbertsen, R. B.; Chan, K.; Schrier, D. J.; Guglietta, A.; Bornemeier, D. A.; Dyer, R. D. J. Med. Chem. 1999, 42, 1161.

18. Vio, L.; Mamolo, M. G.; Laneve, A. Farmaco 1989, 44, 165.

19. Mazzone, G.; Pignatello, R.; Mazzone, S.; Panico, A.; Pennisi, G. Farmaco 1993, 48, 1207.

20. Nomoto, Y.; Takai, H.; Hirata, T.; Teranishi, M.; Ohno, T.; Kubo, K. Chem. Pharm. Bull. 1991, 39, 86.

21. Hatice, N. D.; Arzu, D.; Sevim, R.; Meral, K. U.; Dumrul, C. Bioorg. Med. Chem. 2002, 10, 2893.

22. Wang, X. X.; Li, Zh.; Da, Y. X.; Chang, J. C. Indian J. Chem. 2001, 40B, 422.

23. Palaska, E.; Sahin, G.; Kelicen, P.; Durlu, N. T.; Gulcin Altinok, I. L. Farmaco 2002, 57, 101.

24. Feng, X. M.; Chen, R.; Li, G. Chem. Reag. 1994, 16, 211.

25. (a) Borg, S.; Estenne-Bouhtou, G.; Luthman, K.; Csoregh, I.; Hesselink, W.; Hacksell, U. J. Org. Chem. 1995, 60, 3112. (b) Duncia, J. V.; Santella III, J. B.; Higley, C. A.; VanAtten, M. K.; Weber, P. C.; Alexander, R. S.; Kettner, C. A.; Pruitt, J. R.; Liauw, A. Y.; Quan, M. L.; Knabb, R. M.; Wexler, R. R. Bioorg. Med. Chem. Lett. 1998, 8, 775. (c) Chen, C.; Dagnino, R.; Huang, C. Q.; McCarthy, J. R.; Grigoriadis, D. E. Bioorg. Med. Chem. Lett. 2001, 11, 3165. (d) Jenkins, S. M.; Wadsworth, H. J.; Bromidge, S.; Orlek, B. S.; Wyman, P. A.; Riley, G. J.; Hawkins, J. J. Med. Chem. 1992, 35, 2392.

26. (a) Tsukuda, Y.; Shiratori, M.; Watanabe, H.; Ontsuka, H.; Hattori, K.; Shirai, M.; Shimma, N. Bioorg. Med. Chem. Lett. 1998, 8, 1819. (b) Narayanan, A.; Chapman, D. R.; Upadhyaya, S. P.; Bauer, L. J. Heterocycl. Chem. 1993, 30, 1405.

27. Krakovsky, E. M. D. J.; Rybak, M. J. Pharmacotherapy 1990, 10, 146.

28. Roberts, J.; Schock, K.; Marino, S.; Andriole, V. T. Antimicrob. Agents Chemother. 2000, $44,3381$.

29. (a) Sanati, H.; Belanger, P.; Fratti, R.; Ghannoum, M. Antimicrob. Agents Chemother. 1997, 41, 2492. (b) Espinel-Ingroff, A. J. Clin. Microbiol. 1998, 36, 198.

30. Pfaller, M. A.; Messer, S.; Jones, R. N. Antimicrob. Agents Chemother. 1997, 41, 1124.

31. Stocks, M. J.; Cheshire, D. R.; Reynolds, R. Org. Lett. 2004, 6, 2969.

32. (a) Bordner, C. A. U.S. Patent 2600 689, 1952; Chem. Abstr. 1953, 47, 4373. (b) Friender, W. L.; Andreas, B.; Eckehard, C. Green Chem. 2001, 3, 201. (c) Wayne, W. H.; Matthew, S.; Kevin, T.; Pavitra, K.; Kenneth, G. H.; Christina, M. D.; Richard, B. R.; Thomas, E. P. Bioorg. Med. Chem. Lett. 2006, 16, 3170.

33. (a) Van Tamelen, E. E. J. Am. Chem. Soc. 1951, 73, 3444. (b) Price, C. C.; Kirk, P. F. J. Am. Chem. Soc.1953, 75, 2396. (c) Culvenor, C. C.; Davies, W.; Savige, W. E. J. Chem. Soc. 1952, 4480. (d) Ruccia, M.; Vivona, N.; Cusmano, G. J. Chem. Soc., Chem. Commun., 1974, 358.

34. Padmavathi, V.; Thriveni, P.; Reddy, B. J. M.; Padmaja, A. J. Heterocycl. Chem. 2005, 42, 113. 